\title{
Neuroprotective Effects of Cerebral Ischemic Preconditioning in a Rat Middle Cerebral Artery Occlusion Model: The Role of the Notch Signaling Pathway
}

\author{
Li Chen (iD, 1 Kuan Huang, ${ }^{1}$ Rong Wang, ${ }^{2}$ Qiong Jiang, ${ }^{2}$ Zhenghua Wu, ${ }^{2}$ \\ Weidong Liang, ${ }^{1}$ Rui Guo, ${ }^{1}$ and Lifeng Wang ${ }^{1}$ \\ ${ }^{1}$ First Affiliated Hospital of Gannan Medical University, Ganzhou, Jiangxi 341000, China \\ ${ }^{2}$ Gannan Medical University, Ganzhou, Jiangxi 341000, China \\ Correspondence should be addressed to Li Chen; zgx8778@126.com
}

Received 31 January 2018; Revised 10 May 2018; Accepted 19 June 2018; Published 6 August 2018

Academic Editor: Sung-Chun Tang

Copyright (c) $2018 \mathrm{Li}$ Chen et al. This is an open access article distributed under the Creative Commons Attribution License, which permits unrestricted use, distribution, and reproduction in any medium, provided the original work is properly cited.

Cerebral ischemia-reperfusion (I/R) injury is a major problem worldwide. The Notch signaling pathway plays an important role in neural progenitor cell differentiation and in the inflammatory response after central nervous system injury. This study evaluated whether the neuroprotective effect of cerebral ischemic preconditioning (cIPC) is mediated by the preactivation of the Notch signaling pathway. A rat middle cerebral artery occlusion/reperfusion (MCAO/R) model and glucose deprivation/reoxygenation (OGD/R) cell model were constructed to detect the neuroprotective effects of cIPC. In in vivo experiments, cIPC reduces the neurological functional deficit, cerebral infarction, and cellular apoptosis in the hippocampus induced by middle cerebral artery occlusion/reperfusion (MCAO/R), thus indicating that cIPC can improve neurologic function. Moreover, cIPC can reveal the expression peak of Jagged1, Notch1, NICD, and Hesl protein, thereby indicating that cIPC can preactivate Notch signaling. However, cIPC-induced improvements in neurologic function are compromised by the $\gamma$-secretase inhibitor $\mathrm{N}$-(N-(3,5-difluorophenacetyl)1-alanyl)-S-phenylglycine t-butyl ester (DAPT). In in vitro experiments, OGD preconditioning (OGDPC) can clearly upregulate Notchl expression in the OGD/R-treated neuron and neural stem cell. Notchl pre-overexpression can decrease neuron death and apoptosis under OGD/R treatment. Notchl pre-overexpression can decrease the percentage of G1 stage cells and increase the percentage of S stage cells in OGD/R-treated neural stem cell. Furthermore, Notchl pre-knockdown has the opposite effect on cell survival, apoptosis, and cycle in both OGD/R-treated neuron and neural stem cell. In conclusion, our results demonstrate that the neuroprotective effects of cIPC in a rat $\mathrm{MCAO} / \mathrm{R}$ model are mediated by the preactivation of the Notch signaling pathway.

\section{Introduction}

Cerebral ischemia-reperfusion ( $\mathrm{I} / \mathrm{R})$ injury is a major problem worldwide. Cerebral I/R injury often causes irreversible brain damage that leads to functional impairment and/or neuronal death $[1,2]$. The treatment for this disorder remains palliative only. Currently, this illness has no effective pharmacotherapy. Ischemic preconditioning (IPC), elicited by a nonfatal brief occlusion of blood flow, is a technique for producing resistance to the loss of blood supply. Emerging data from clinical trials have shown that IPC is a therapeutic strategy against subsequent fatal ischemic insults in vital organs (e.g., kidney and heart) [3-7]. However, cerebral IPC
(cIPC), a transient sublethal cerebral ischemia, cannot be applied in clinic because of security and ethical concerns. Therefore, elucidating the regulating mechanism of cIPC is important to discover drugs that behave in a similar way.

Notch signaling is evolutionarily conserved from Drosophila to humans. It plays an important role in neural stem cell maintenance and neurogenesis in the embryonic brain and adult brain $[8,9]$. During neocortical development, Notch signaling inhibits neuronal differentiation and maintains the neural stem/progenitor cell pool to enable successive waves of neurogenesis, which are followed by gliogenesis [9]. Aside from its role in neural development, Notch signaling 
also has a role in cerebral I/R injury [3, 10]. The Notch signaling pathway is important in neural progenitor cell differentiation and in the inflammatory response after central nervous system injury [11]. A recent study showed that the neuroprotective effects of isoflurane preconditioning in a murine transient global cerebral I/R model are mediated by the preactivation of the Notch signaling pathway [12, 13]. However, whether the Notch signaling pathway plays a role in the neuroprotective effects of cIPC remains unclear.

No clinical data are available on the neuroprotective effect of cIPC. Therefore, the function and the regulating mechanism of cIPC are only studied in animal models, such as the middle cerebral artery occlusion (MCAO) model. In this study, we investigated the relationship between changes in the Notch signaling pathway and the neuroprotection induced by CIPC in vivo and in vitro. Our hypothesis is that the neuroprotection of cIPC is mediated by the preactivation of the Notch signaling pathway.

\section{Materials and Methods}

2.1. Experimental Animals. Ninety male Sprague-Dawley (SD) rats (200-220 g, 9 weeks old) were obtained from Vital River Laboratories (Beijing, China). The animals were allowed free access to food and water under controlled room temperature $\left(25^{\circ} \mathrm{C}\right)$ and lighting (14:10 light/dark cycle) conditions for this study. All animal experimental procedures were approved by the Institute of Animal Care and Use Committee of First Affiliated Hospital of Gannan Medical College and performed in accordance with the Guide for the Care and Use of Laboratory Animals (NIH publication no. 85-23, National Academy Press, Washington, DC, USA, revised 1996).

2.2. MCAO/Reperfusion (MCAO/R) and cIPC Model. The MCAO model was performed as described previously [14], with minor modifications. Briefly, the rats were placed in the supine position, with the limbs taped to the operating table, after they were anesthetized with an intraperitoneal injection of $350 \mathrm{mg} / \mathrm{kg}$ chloral hydrate. After a midline skin incision, the left external carotid artery was exposed, and its branches were ligated. A nylon monofilament (diameter $=0.26 \mathrm{~mm}$ ) coated with silicon was introduced into the internal carotid artery through the common carotid artery and advanced until faint resistance was felt. After 90 min of occlusion, blood flow was restored by withdrawing the nylon thread to allow reperfusion. Sham-operated control rats received the same procedure except filament insertion. The cIPC operation was the same as the MCAO, but the occlusion was conducted for $10 \mathrm{~min}$. The rectal temperature was maintained at $37 \pm 0.5^{\circ} \mathrm{C}$ by lamp irradiation. All surgical procedures were performed under sterile conditions.

2.3. Experimental Groups. Ninety rats were randomly allocated to six groups: sham group: a healthy control group that underwent sham surgery; cIPC group: an MCAO/R model group that received $10 \mathrm{~min}$ of ischemia and reperfusion for one day; $\mathrm{MCAO} / \mathrm{R}$ group: an MCAO model group that received $90 \mathrm{~min}$ of ischemia and reperfusion for $2 \mathrm{~h}$ for one or seven days; cIPC+ MCAO/R group: rats initially received $10 \mathrm{~min}$ of ischemia after one day of reperfusion and then received $90 \mathrm{~min}$ of ischemia and reperfusion for $2 \mathrm{~h}$ for one or seven days; N-[N-(3,5-difluorophenacetyl)l-alanyl]-S-phenylglycine tert-butyl ester (DAPT) + cIPC $+\mathrm{MCAO} / \mathrm{R}: 100 \mathrm{mg} / \mathrm{kg} \gamma$-secretase inhibitor N-(N-(3,5-difluorophenacetyl)-1-alanyl)-S-phenylglycine t-butyl ester (DAPT) was injected intraperitoneally $3 \mathrm{~h}$ before cIPC+ $\mathrm{MCAO} / \mathrm{R}$ surgery; and $\mathrm{DMSO}+\mathrm{cIPC}+\mathrm{MCAO} / \mathrm{R}$ group: equal volume vehicle DMSO was injected intraperitoneal injection $3 \mathrm{~h}$ before cIPC+ MCAO/R surgery.

2.4. Neurological Function Testing. Neurological functional scores were assessed $24 \mathrm{~h}$ postoperatively using Longa's method [14]. Neurological status was scored on a four-point scale as follows: $0=$ no neurological symptoms; 1 point $=$ inability to completely extend the right front paw; 2 = rotating while crawling and falling to the right side; $3=$ inability to walk without assistance; and $4=$ unconscious. Rats scoring 1-3 points met the inclusion criteria for the experiment, and those scoring 0 or 4 were excluded.

2.5. Measurement of the Cerebral Infarction Volume. After neurological function testing, the rats were killed for infarction volume analysis. Whole brains were quickly removed and frozen at $20^{\circ} \mathrm{C}$ for $30 \mathrm{~min}$. The brains were cut into five coronal sections. The first cut was at the midpoint between the anterior pole and the optic chiasm. The slices were stained in $0.5 \%$ triphenyltetrazolium chloride (TTC) solution at a temperature of $37^{\circ} \mathrm{C}$ for $15 \mathrm{~min}$ away from light and then fixed with $4 \%$ paraformaldehyde for $4 \mathrm{~h}$. A digital camera and an image analysis system were used for the image acquisition and analysis. The infarct size was determined using Image Pro-Plus 6.0 software (Media Cybernetics, Silver Springs, MD, USA). Infarct ratio $(\%)=$ the calibrated infarct volume/volume of the contralateral hemisphere.

2.6. Terminal Deoxynucleotidyl Transferase-Mediated $2^{\prime}$ Deoxyuridine 5'-Triphosphate Nick-End Labeling (TUNEL) Assay for Apoptotic Nuclei. For each rat, six sections were analyzed using the In Situ Cell Death Detection Kit, AP (Roche Molecular Biochemicals, Mannheim, Germany) according to the manufacturer's protocols. For image analysis, three random fields $(200 \times)$ from each rat were observed for TUNEL-positive cells using fluorescence microscopy (Carl Zeiss, Jena, Germany).

2.7. Neuron and Neural Stem Cell (NSC) Isolation. Hippocampal neuron was isolated from specific pathogen-free grade fetal SD rats (14 days, Vital River Laboratories) and cultured as described previously [15]. The isolated neuron was identified by detecting the expression of MAP2 and GFAP using immunofluorescence as described previously [16]. NSC was isolated from specific pathogen-free grade fetal SD rats (14 days, Vital River Laboratories) as described previously [16]. Isolated NSC was identified by detecting the expression 
of nestin and BrdU using immunofluorescence as described previously [16].

2.8. Notch1 Overexpression and Knockdown by AdenovirusMediated Transduction. The shRNA sequence targeting GCAAUAAGGUCUGCAACCU and the full open reading frame of Notch1 were cloned into a linearized adenovirus plasmid GV314 (Genechem) with T4 DNA ligase and transfected into competent Escherichia coli cells. Positive clones were selected by ampicillin resistance and then sequenced. The Notch1 overexpression adenovirus (Ad-Notch1) and Notchl shRNA adenovirus (Ad-sh-Notch1) were packaged in HEK293T cells and purified with an Adeno- $\mathrm{X}^{\mathrm{TM}}$ Virus Purification Kit (BD Biosciences, San Jose, CA, USA). The endpoint dilution method was used to determine the viral titer. Adenovirus particles expressing a scrambled sequence (Ad-NC; purchased from Genechem) served as the negative control. For cell infection, the total NSC and neuron were seeded in six-well plates with or without coverslips. After $24 \mathrm{~h}$, the virus was added to the cells at a multiplicity of infection of 100 in the presence of $4 \mu \mathrm{g} / \mathrm{ml}$ polybrene. Cells not transfected with the virus were classified as MOCK. Twenty-four hours after infection, the medium was changed to complete DMEM and then changed every three days. After $72 \mathrm{~h}$, the viral infection efficiency was assessed under fluorescence microscopy. Cells were harvested for experiments on day 8 after infection.

2.9. Cell Model and Experimental Group. Sham: cells were cultured in a complete medium at $37^{\circ} \mathrm{C}$ in a humidified $5 \% \mathrm{CO}_{2}$ incubator. OGD/R injury model: cells were cultured in a sugar-free and serum-free culture medium in a Billups-Rothenberg chamber with $94 \% \mathrm{~N}_{2}, 1 \% \mathrm{O}_{2}$, and $5 \%$ $\mathrm{CO}_{2}$ at $37^{\circ} \mathrm{C}$ for $2 \mathrm{~h}$ and then cultured in a complete medium at $37^{\circ} \mathrm{C}$ in a humidified $5 \% \mathrm{CO}_{2}$ incubator for $24 \mathrm{~h}$. OGDPC model: cells were cultured in a sugar-free and serum-free culture medium in a Billups-Rothenberg chamber with $94 \%$ $\mathrm{N}_{2}, 1 \% \mathrm{O}_{2}$, and $5 \% \mathrm{CO}_{2}$ at $37^{\circ} \mathrm{C}$ for $20 \mathrm{~min}$ and then cultured in a complete medium at $37^{\circ} \mathrm{C}$ in a humidified $5 \% \mathrm{CO}_{2}$ incubator for $20 \mathrm{~min}$. OGDPC+ OGD/R model: cells were cultured in the same condition as in the OGDPC model and then cultured in the same condition as in the OGD/R model.

2.10. Western Blotting. The total protein of the hippocampal tissue or cells was isolated using lysis buffer containing protease inhibitors, and the protein concentration of each sample was estimated by the Lowry method using a protein assay kit. Aliquots of lysates containing $20 \mathrm{lg}$ of protein were separated by electrophoresis on $8 \%$ gradient sodium dodecyl sulfate-polyacrylamide gels (SDS-PAGE) and transferred to nitrocellulose membranes (Bio-Rad Laboratories, Richmond, CA, USA). After blocking for $1 \mathrm{~h}$ at room temperature with $5 \%(\mathrm{w} / \mathrm{v})$ skim milk powder, blots were incubated overnight at $4^{\circ} \mathrm{C}$ with the primary antibodies anti-Jagged1, anti-Notch1, anti-NICD, and anti-Hesl (Abcam, Cambridge, MA, USA). Anti-GAPDH antibody was used as the internal control. Anti-rabbit antibody was used as the secondary antibody, and antigens were detected using the standard chemiluminescence method (ECL; Millipore Biotech, Billerica, MA, USA).

2.11. Cell Proliferation Assays. Cell proliferation was measured using the CellTiter 96 AQueous One Solution Cell Proliferation Assay Kit (Promega, Madison, WI, USA) according to the manufacturer's protocol. Adenovirus-transduction NSC and neuron cells $\left(1 \times 10^{4}\right)$ were seeded onto a 96-well plate. The cells were exposed to a hypoxia condition and cultured in a Billups-Rothenberg chamber with $94 \% \mathrm{~N}_{2}, 1 \%$ $\mathrm{O}_{2}$, and $5 \% \mathrm{CO}_{2}$ at $37^{\circ} \mathrm{C}$ for $2 \mathrm{~h}$. Subsequently, the cells were maintained at $37^{\circ} \mathrm{C}$ in a humidified $5 \% \mathrm{CO}_{2}$ incubator for another 24,48 , or $72 \mathrm{~h}$. After culturing, $10 \mu \mathrm{L}$ of the CellTiter 96 AQueous One Solution reagent was added to each well. The cells were then incubated for $4 \mathrm{~h}$ at $37^{\circ} \mathrm{C}$. Absorbance was measured at $490 \mathrm{~nm}$ using a microplate reader (Multiskan MK3, Thermo Scientific, Vantaa, Finland). The survival rate was calculated using the following formula: survival rate $=$ $\left(\mathrm{OD}_{\text {test }} / \mathrm{OD}_{\text {negative control }}\right) \times 100 \%$.

2.12. Flow Cytometry Analysis. Adenovirus-transduction NSC and neuron cells $\left(2 \times 10^{5}\right)$ cells were seeded onto a 24 -well plate. The cells were exposed to a hypoxia condition, cultured in a Billups-Rothenberg chamber with $94 \%$ $\mathrm{N}_{2}, 1 \% \mathrm{O}_{2}$, and $5 \% \mathrm{CO}_{2}$ at $37^{\circ} \mathrm{C}$ for $2 \mathrm{~h}$. Subsequently, the cells were maintained at $37^{\circ} \mathrm{C}$ in a humidified $5 \% \mathrm{CO}_{2}$ incubator for reperfusion. After reperfusion for $24 \mathrm{~h}$, the cells were harvested for cell apoptosis and cell cycle analysis. Annexin V-FITC apoptosis detection and cell cycle detection kits were used to analyze the apoptosis rate and the cell cycle distribution according to the manufacturer's protocols (Keygen, Nanjing, China). The percentage of apoptotic cells and the cell cycle distribution were analyzed by flow cytometry (BD Biosciences, San Jose, CA, USA). Each experiment was repeated three times.

2.13. Statistical Analysis. All data were expressed as mean \pm SD. Statistical analyses were performed using the Statistical Package for the Social Sciences (SPSS) v19.0 software (SPSS Inc., Chicago, IL, USA). We compared the groups using one-way ANOVA, followed by post hoc tests of least significant difference (LSD) for multiple pairwise comparisons. A value of $p<0.05$ was considered statistically significant.

\section{Results}

3.1. cIPC Reduced the Neurological Functional Deficit and Cerebral Infarction Induced by MCAO/R. After reperfusion for one day, neurological function testing was performed to examine the neurological deficit score of each rat group. The results showed that cIPC had no obvious effect on the neurological deficit score unlike in the sham group (Figure 1(a)). The neurological deficit score of the MCAO/R group was significantly higher than that of the sham group (Figure 1(a)). However, this increase could be decreased by cIPC (Figure 1(a)). The results of TTC staining showed no 


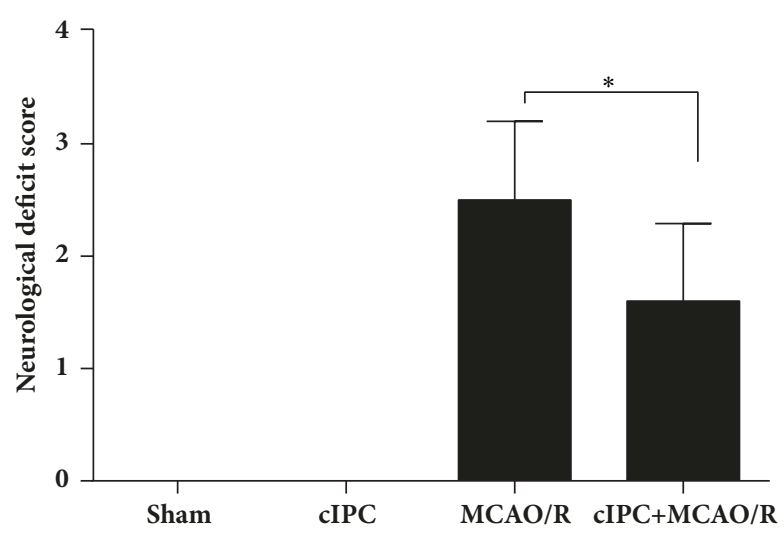

(a)

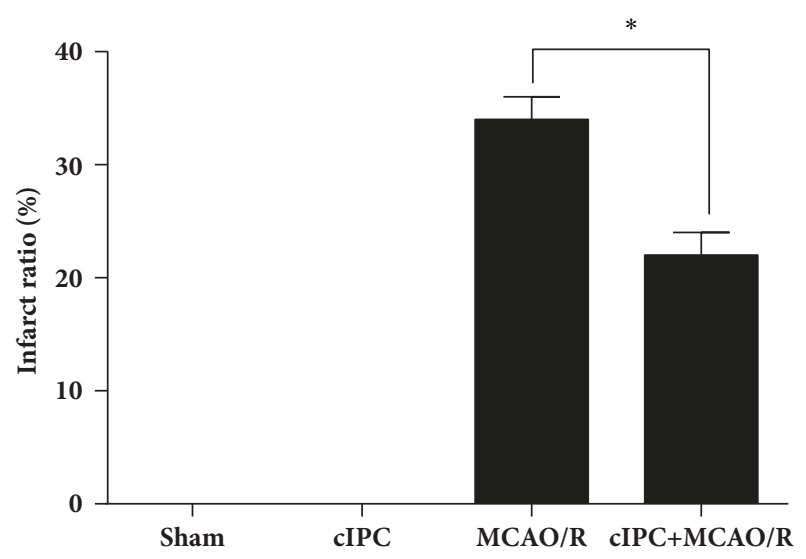

(b)



(c)

FIGURE 1: cIPC reduced the neurological functional deficit and cerebral infarction induced by MCAO/R for $24 \mathrm{~h}$. (a) The neurological functional deficit score was examined to evaluate the effect of cIPC on the neurological functional deficit induced by MCAO/R. (b) TTC staining was conducted to evaluate the effect of cIPC on cerebral infarction induced by MCAO/R. (a) is the infarct ratio calculated according to the results of TTC staining. (c) is the represented image of TTC staining. ${ }^{*} p<0.05$.

cerebral infarction in both sham and cIPC groups (Figures $1(\mathrm{~b})$ and $1(\mathrm{c}))$. However, the infarct ratio of the $\mathrm{MCAO} / \mathrm{R}$ group was significantly higher than that of the sham group, and this increase could be decreased by cIPC (Figures 1(b) and $1(\mathrm{c}))$.

\section{2. cIPC Reduced Cellular Apoptosis in the Hippocampus} Induced by $M C A O / R$. After reperfusion for one day, the total brains of each rat group were obtained for TUNEL assay for apoptotic nuclei. The apoptotic nuclei in the hippocampus were analyzed. As shown in Figure 2, no obvious difference was found in the number of apoptotic nuclei in the hippocampus of rats in the sham and cIPC groups, thus indicating that cIPC could not increase the number of apoptotic cells. The number of apoptotic nuclei in the hippocampus of rats in the $\mathrm{MCAO} / \mathrm{R}$ group significantly increases compared with that in the sham group, and this increase could be decreased by cIPC.

3.3. Effect of cIPC on the MCAO/R-Induced Notch Signaling Activation. To investigate the possible mechanism of the neuroprotective effects of cIPC, we detected the expression levels of Jagged1, Notchl, NICD, and Hesl, which belong to the Notch signaling pathway, in the hippocampus. Western blot analyses showed that cIPC clearly increased the expressions of Jaggedl, Notchl, NICD, and Hesl protein unlike in the sham groups (Figures 3(a), 3(b), 3(c), 3(d), and 3(e)). $\mathrm{MCAO} / \mathrm{R}$ also increased the expressions of Jagged1, Notchl, NICD, and Hesl protein unlike in the sham group $2 \mathrm{~h}, 1$ day, and 7 days after reperfusion, and the peak of these increases occurred one day after reperfusion (Figures 3(a), 3(b), 3(c), 3(d), and 3(e)). At the same time point, the 

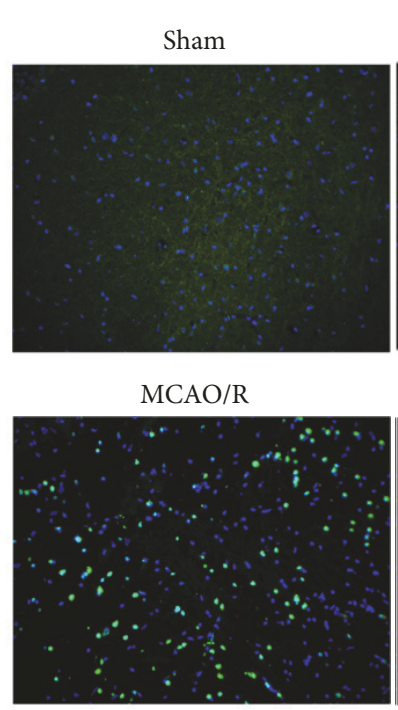

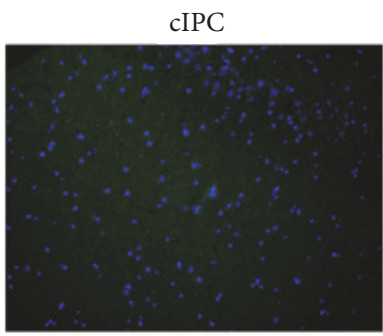

cIPC+MCAO/R

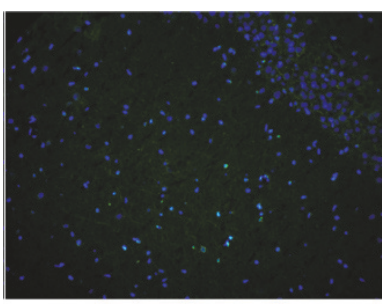

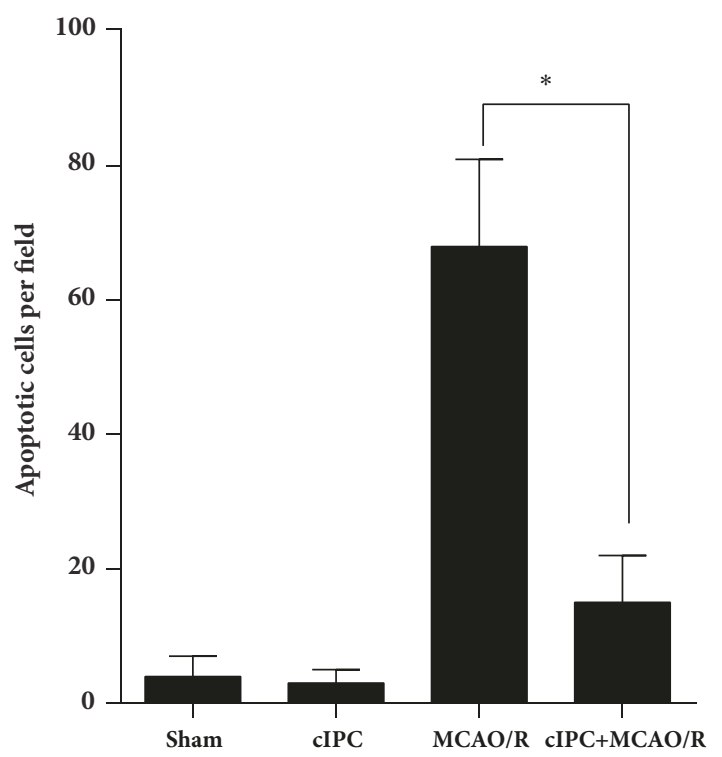

FIGURE 2: cIPC reduced cell apoptosis in the hippocampus induced by MCAO/R for $24 \mathrm{~h}$. TUNEL assay was conducted to evaluate the effect of cIPC on the hippocampus cell apoptosis induced by MCAO/R. On the left is the represented image of the TUNEL assay (200×). The blue color is cell nucleus stained by DAPI. Green color is TUNEL-positive cells. On the right is the statistical result of the number of apoptotic cells. ${ }^{*} p<0.05$.

expressions of Jagged1, Notch1, NICD, and Hes1 protein in the $\mathrm{CIPC}+\mathrm{MCAO} / \mathrm{R}$ group were higher than those in the $\mathrm{MCAO} / \mathrm{R}$ group, thus indicating that cIPC could advance the expression peaks of Jagged1, Notch1, NICD, and Hesl protein (Figures 3(a), 3(b), 3(c), 3(d), and 3(e)). All these results indicated that cIPC could preactivate the Notch signaling.

\section{4. cIPC-Induced Improvements in Neurologic Function Are} Compromised by $\gamma$-Secretase Inhibitor DAPT. To further determine the role of Notch signaling in the cIPC-induced neuroprotective effects in the rat middle $\mathrm{MCAO} / \mathrm{R}$ model, $\gamma$-secretase inhibitor DAPT or vehicle DMSO was injected intraperitoneally $3 \mathrm{~h}$ before cIPC. The results of Western blot showed that Jagged1, Notch1, NICD, and Hesl expression levels were decreased in the brain tissues of DAPT-treated rats (Figure 4(a)), indicating that Notch signaling was successfully blocked by DAPT pretreatment. The results of the neurological function testing showed that DAPT pretreatment could increase the neurological deficit score, which was decreased by cIPC in the rat MCAO/R model (Figure 4(b)). In addition, the results of TTC staining showed that DAPT pretreatment could increase the infarct ratio, which was decreased by cIPC in the rat middle MCAO/R model (Figures $4(\mathrm{c})$ and $4(\mathrm{~d})$ ). DAPT pretreatment could also increase the number of apoptotic nuclei in the hippocampus decreased by cIPC in the rat middle MCAO/R model (Figure 5).

\subsection{Effect of OGDPC on Notch1 Expression in $O G D / R$ -} Treated Neuron and NSC. MAP2 and GFAP were highly expressed in the isolated hippocampal neuron (Figure 6(a)). Nestin and BrdU were highly expressed in isolated NSC (Figure 6(b)). These results indicated that the hippocampal neuron and NSC were successfully isolated and could be used for the subsequent assays. Then, the cell models of sham, OGDPC, OGD/R, and OGDPC + OGD/R were constructed. The Western blotting results showed that OGDPC and/or OGD/R treatment could upregulate the protein expression of Notchl in both neuron and neural stem cell (Figures 6(c) and 6(d)). In addition, the Notch1 expression induced by OGDPC + OGD/R was higher than that induced by $\mathrm{OGD} / \mathrm{R}$ only at the same time point after reperfusion, thereby indicating that OGDPC could preactivate Notch signaling.

\subsection{Effect of Notch1 Overexpression and Silencing on OGD/R-} Treated Neuron Cell Survival and Cellular Apoptosis. After transfection with Ad-NC, Ad-Notchl, and Ad-sh-Notchl for three days, the expression level of Notchl in neuron was detected using Western blotting. The results showed that Notchl was successfully knockdown after transfected with Ad-sh-Notchl and successfully overexpressed after transfection with Ad-Notch1 (Figure 7(a)). Then, the effect of Notch1 overexpression and silencing on OGD/R-treated neuron cell survival and cellular apoptosis was detected using cell proliferation assays and flow cytometry analysis. The results showed that the survival rate of a neuron transfected with Ad-Notchl was higher than that transfected with Ad-NC one, two, and three days after OGD/R treatment (Figure 7(b)). The percentage of apoptotic neuron transfected with Ad-Notch1 was significantly lower than that transfected with Ad-NC $2 \mathrm{~h}$ after OGD/R treatment (Figures 7(c) and 7(d)). These results indicated that Notch1 pre-overexpression could decrease neuron death and apoptosis under OGD/R treatment. In addition, Notchl pre-knockdown could advance neuron death and apoptosis under OGD/R treatment (Figures 7(b), $7(\mathrm{c})$, and $7(\mathrm{~d}))$. 


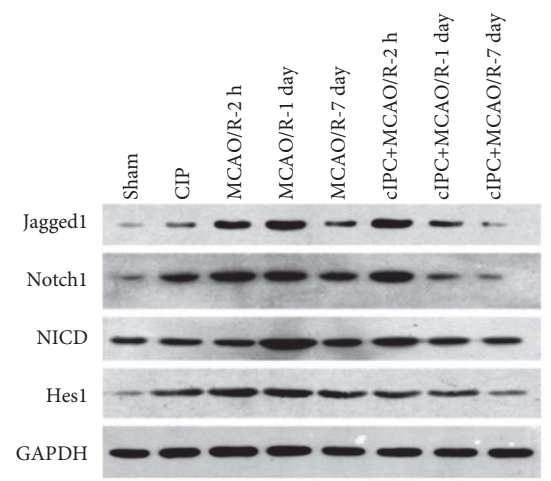

(a)

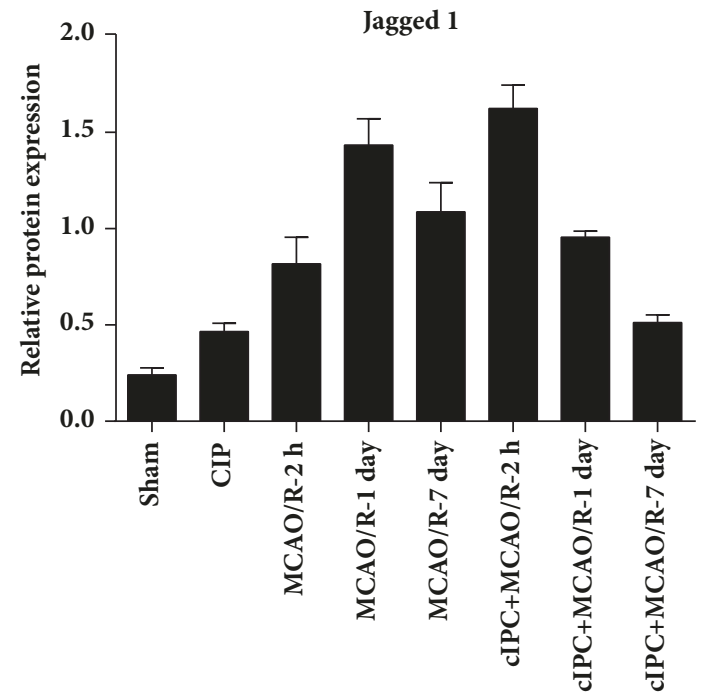

(b)

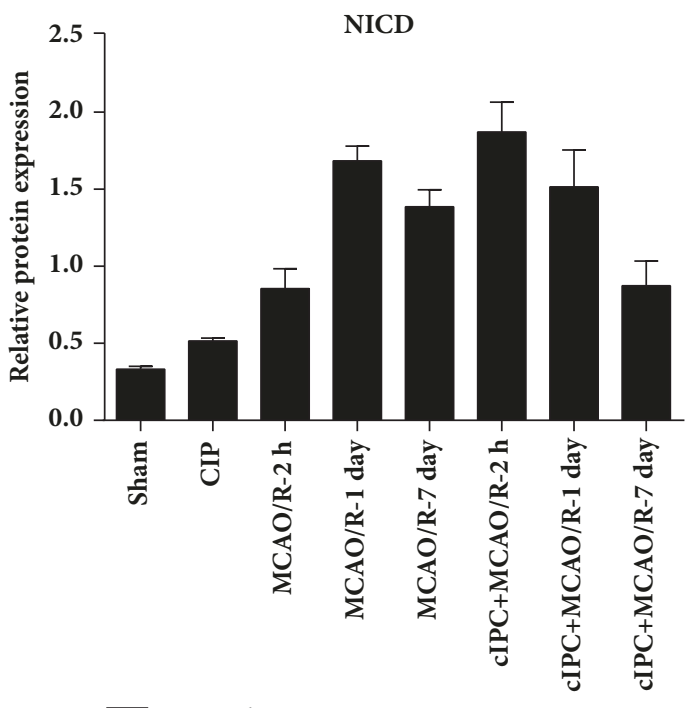

(d)

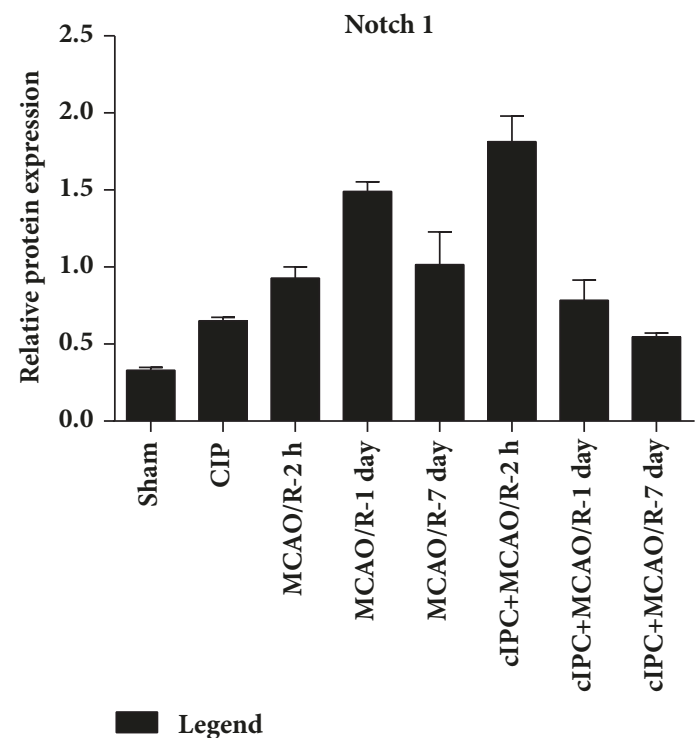

(c)

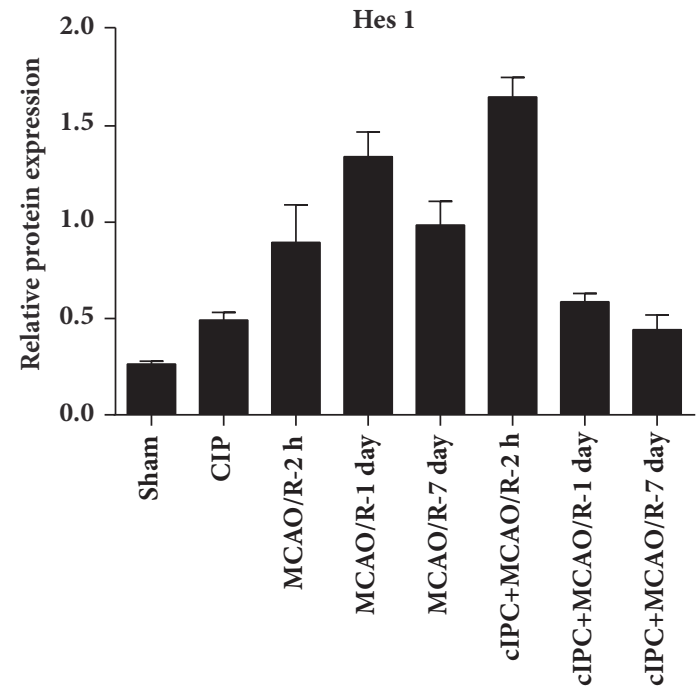

(e)

FIGURE 3: cIPC could advance the expression peak of Jagged1, Notch1, NICD, and Hes1 protein in the hippocampus of the rat (MCAO/R) model. (a) The represented images. (b-e) Relative protein level of Jagged 1, Notch1, NICD, and Hes1 expressed as the relative ratio of density of the target protein to the reference protein GAPDH. 

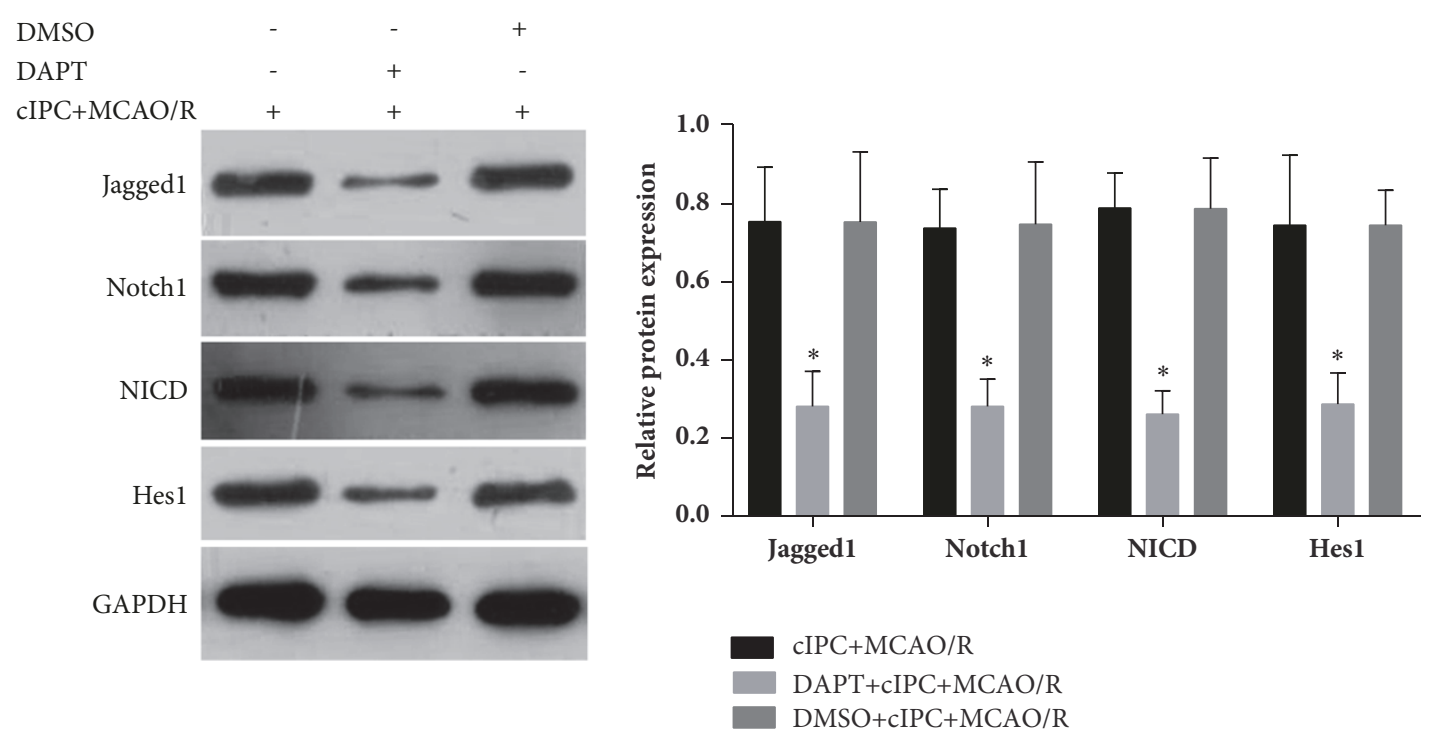

(a)

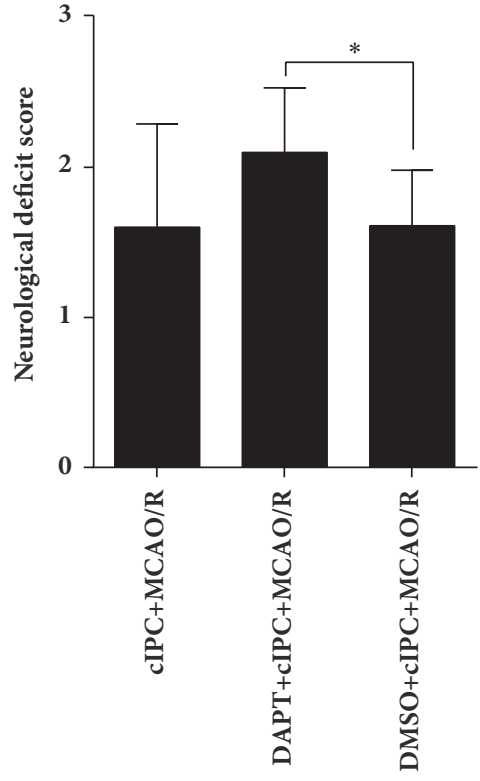

(b)

$\mathrm{DMSO}+\mathrm{cIPC}+\mathrm{MCAO} / \mathrm{R}$

$\mathrm{DAPT}+\mathrm{cIPC}+\mathrm{MCAO} / \mathrm{R}$

$\mathrm{cIPC}+\mathrm{MCAO} / \mathrm{R}$

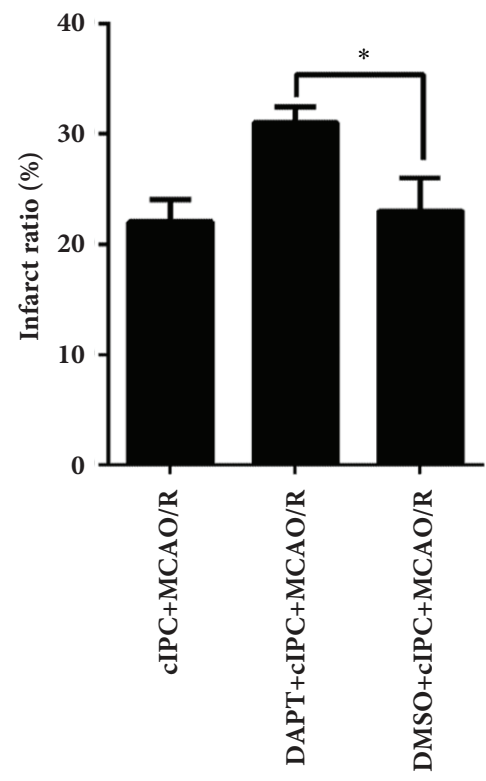

(c)

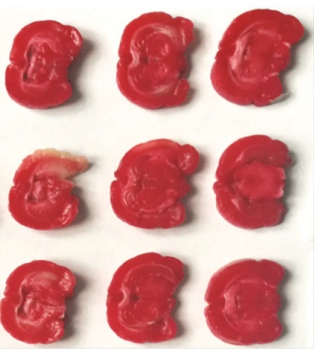

(d)

FIGURE 4: The cIPC-induced improvements in the neurological functional deficit and cerebral infarction induced by MCAO/R are compromised by the $\gamma$-secretase inhibitor DAPT. (a) Jagged1, Notch1, NICD, and Hesl expression levels in the brain tissues of DAPT-treated rats. (b) The neurological functional deficit score was examined to evaluate the effect of DAPT and/or cIPC on the neurological functional deficit induced by MCAO/R. (c-d) TTC staining was conducted to evaluate the effect of DAPT and/or cIPC on cerebral infarction induced by MCAO/R. The top figure is the infarct ratio calculated according to the results of TTC staining (c). The bottom photo is the represented image of TTC staining (d). ${ }^{*} p<0.05$. 

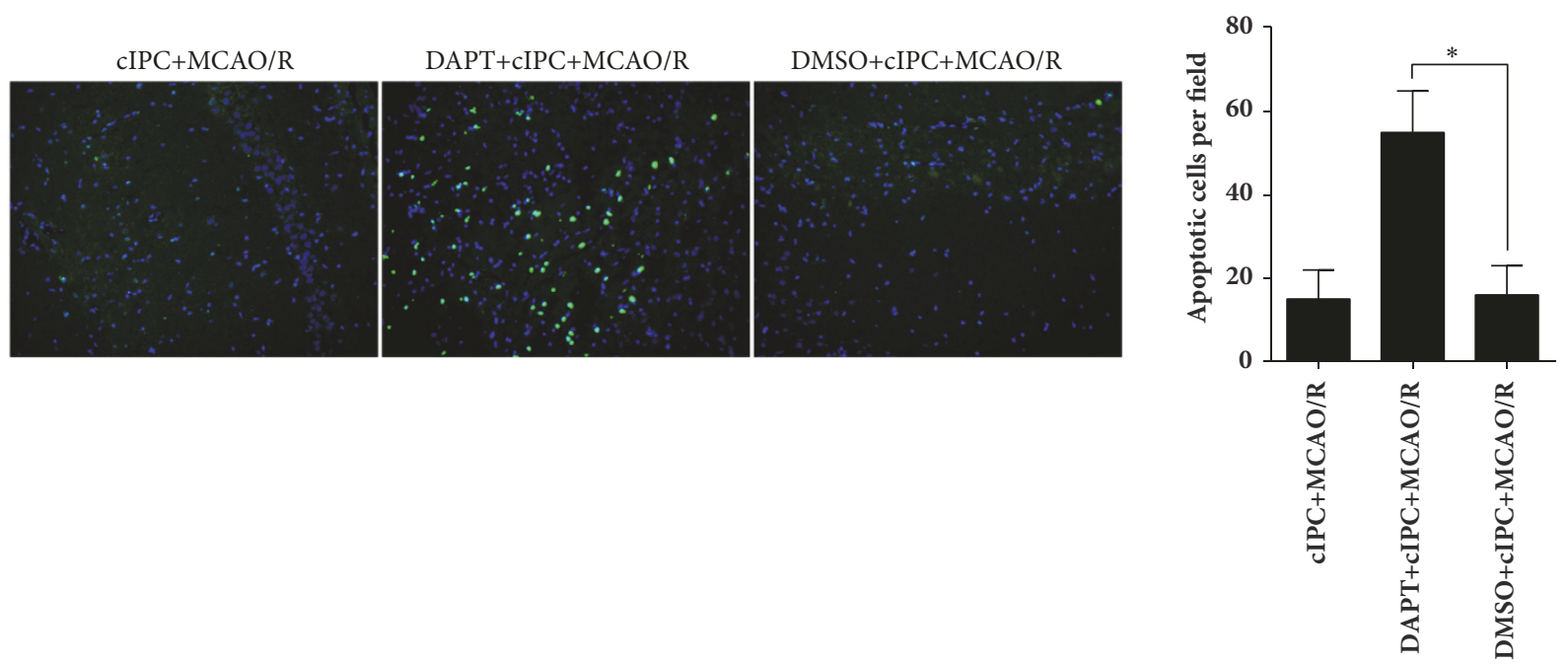

FIGURE 5: cIPC reduced cell apoptosis in the hippocampus induced by MCAO/R for $24 \mathrm{~h}$. TUNEL assay was conducted to evaluate the effect of DAPT and/or cIPC on the hippocampus cell apoptosis induced by MCAO/R. The photo on the left is the represented image of TUNEL assay $(200 \times)$. The figure on the right is the statistical result of the number of apoptotic cells. ${ }^{*} p<0.05$.

3.7. Effect of Notch1 Overexpression and Silencing on the OGD/R-Treated NSC Cell Cycle. After transfection with AdNC, Ad-Notch1, and Ad-sh-Notch1 for three days, the expression level of Notchl in neural stem cells was detected using Western blotting. The results showed that Notch1 was successfully knocked down after transfection with Adsh-Notchl and successfully overexpressed after transfection with Ad-Notch1 (Figure 8(a)). Then, the effect of Notch1 overexpression and silencing on OGD/R-treated neuron cell cycle was detected using flow cytometry analysis. The results showed that the percentage of G1 stage cells decreased after transfection with Ad-Notchl and increased after transfection with Ad-sh-Notch1 (Figures 8(b) and 8(c)). In addition, the percentage of $S$ stage cells increased after transfection with Ad-Notchl and decreased after transfection with Ad-shNotch1 (Figures $8(\mathrm{~b})$ and $8(\mathrm{c})$ ). These results indicated that Notchl pre-overexpression could promote neuron stem cell division.

\section{Discussion}

Cerebral I/R injury induced by irreversible functional impairment and/or neuronal death is a controversial issue in the treatment of cerebral I/R injury-related diseases, such as stroke and cerebral trauma $[1,2]$. Therefore, developing an effective therapy to reduce the harm of cerebral I/ $\mathrm{R}$ is critical. cIPC is one potential treatment option, but it cannot be applied clinically because of security and ethical concerns. Further illuminating the therapeutic effect and the regulating mechanism of cIPC is important to discover drugs that behave in similar ways. The present study demonstrates that $\mathrm{cIPC}$ has a neuroprotective effect in the rat $\mathrm{MCAO} / \mathrm{R}$ model. Moreover, cIPC induces the preactivation of the Notch signaling pathway, and the neuroprotection induced by CIPC is reduced by the administration of the Notch signaling inhibitor DAPT prior to cIPC. Our study provides theoretical basis for the molecular mechanism of cIPC and for the targeted therapy of cerebral I/R injury-related diseases.

Our result shows that cIPC significantly inhibits cerebral infarction and cellular apoptosis and improves the neurological functional deficit, in the hippocampus induced by $\mathrm{MCAO} / \mathrm{R}$. This result indicates that $\mathrm{CIPC}$ has a neuroprotective effect in the rat MCAO/R model. The MCAO model is a reliable method for studying reversible regional ischemia [14]. Therefore, our model demonstrates that cIPC has a neuroprotective on lethal ischemic insult. This result is consistent with those of previous studies, and it indicates that our model can be used to examine the mechanism of cIPC.

Although significant progress has been made toward identifying some of the major molecules involved in the neuroprotection of cIPC, the mechanisms behind the formation of cIPC protection remain largely unknown [17]. Notch signaling plays a key role in brain physiology and pathology $[8,18,19]$. However, its role in the formation of cIPC protection remains unclear. Our results in vivo and in vitro will fill the current biological gap. First, we found that cIPC could advance the expression peak of Jagged1, Notch1, NICD, and Hes1 protein in the cIPC+MCAO/R group unlike in the $\mathrm{MCAO} / \mathrm{R}$ group. Jagged1 is the ligand for the receptor Notch1 [20]. Hes1 is a major transcriptional downstream regulator of the Notch signaling pathway [21]. Therefore, cIPC can certainly preactivate the Notch signaling. Second, we found that the cIPC-induced improvements in neurologic function are compromised by the $\gamma$-secretase inhibitor DAPT. Third, Notchl pre-knockdown can advance primary neuron death and apoptosis under I/R treatment. Fourth, Notchl preoverexpression can promote neuron stem cell division under $\mathrm{I} / \mathrm{R}$ treatment. These results reveal that Notch signaling is involved in the neuroprotective effects of cIPC in the rat $\mathrm{MCAO} / \mathrm{R}$ model. However, our present result seems to contradict some previous public reports. There are many reports 


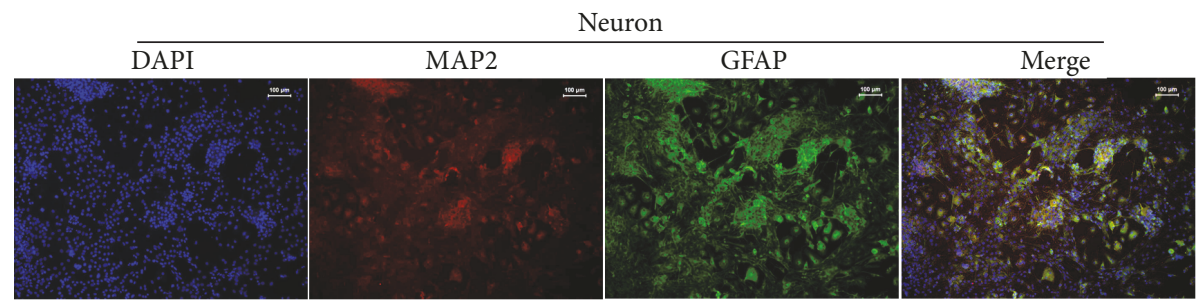

(a)

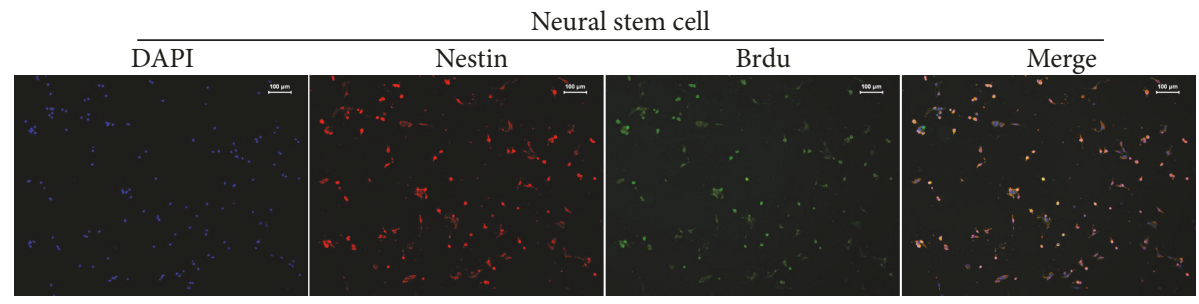

(b)
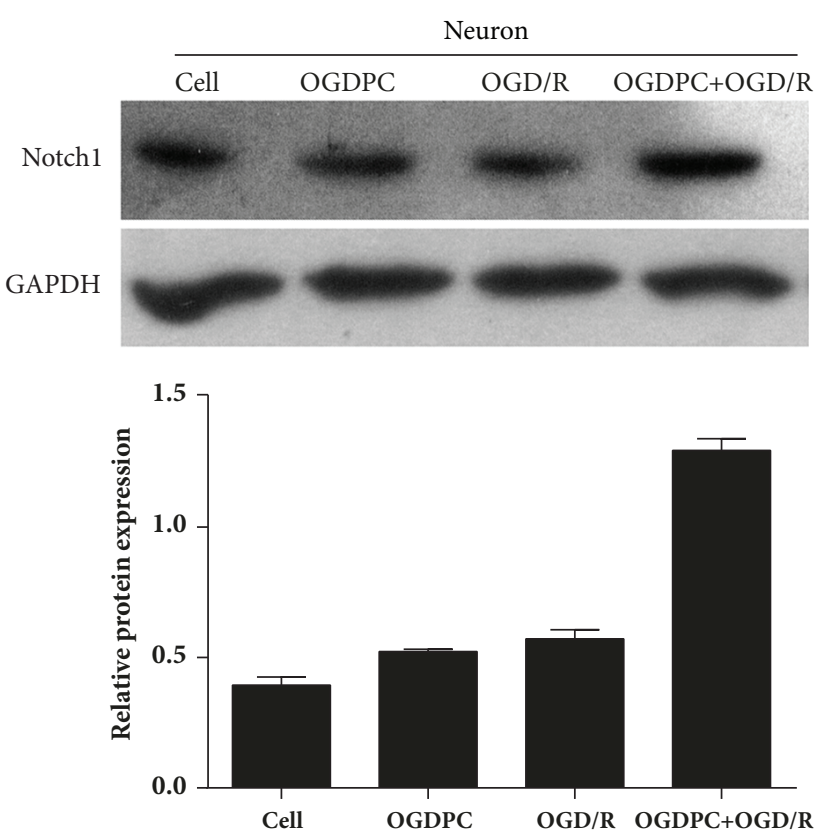

(c)
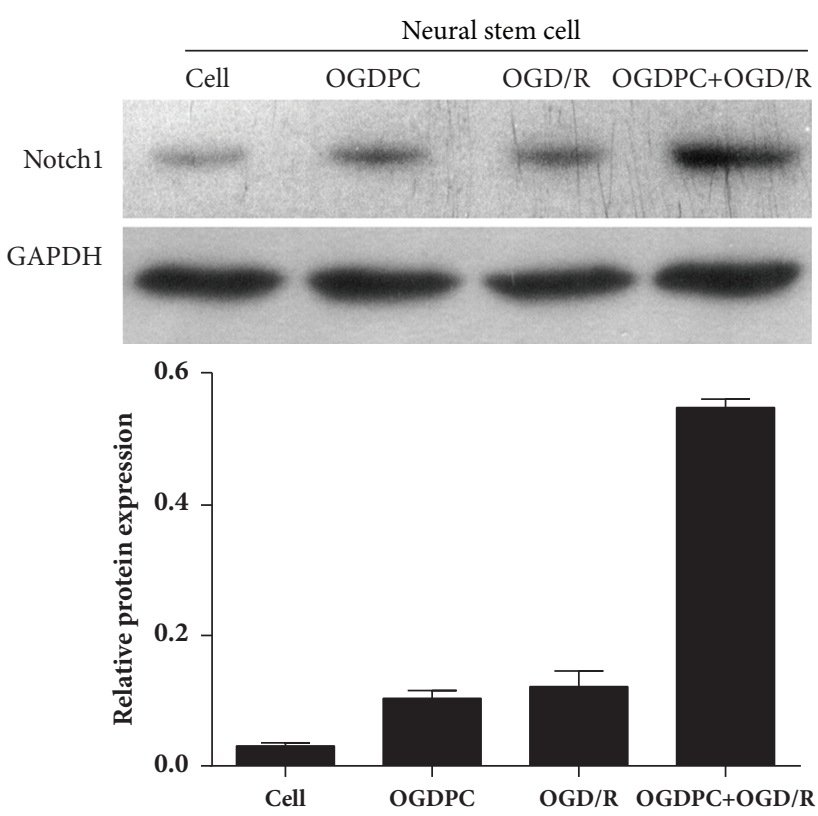

(d)

FIGURE 6: Effect of OGDPC on Notch1 expression in OGD/R-treated neuron and neural stem cell. (a) The isolated neuron was identified by detecting the expression of MAP2 and GFAP using immunofluorescence. (b) Isolated NSC was identified by detecting the expression of nestin and BrdU using immunofluorescence. (c-d) Expression levels of Notch1 in the neuron (c) or neural stem cell (d) models of sham, OGDPC, $\mathrm{OGD} / \mathrm{R}$, and OGDPC + OGD/R.

showing detrimental function of Notch signaling in ischemic stroke [19, 22-25]. It is sure that Notchl signaling pathway can be activated by cerebral ischemia and hypoxia. However, the function of the downstream genes regulated by Notch1 signaling pathway and four prominent interacting pathways (NF- $\kappa$ B, p53, HIF-1 $\alpha$, and Pin1) is complex [26]. So we predict that Notch1 signaling pathway may play different role in different physiological environment by regulated the expression of different downstream genes. Our predication may be partly supported by some previous studies. For example, the preactivation of the Notch signaling pathway contributes to the neuroprotective effects of isoflurane preconditioning in a murine transient global cerebral I/R model [12], and the activation of the canonical Notch signaling pathway is involved in the ischemic tolerance induced by sevoflurane preconditioning in a mouse $\mathrm{MCAO} / \mathrm{R}$ model [27]. So the role of Notchl signaling pathway in cerebral I/R injury-related diseases is still inconclusive and more study is needed to carry out.

Although Notch signaling is certain to be involved in cerebral I/R injury $[10,12,18]$, no direct evidence demonstrates the role of Notch signaling in the neuroprotective effects of cIPC. Therefore, our results provide further evidence to confirm the role of Notch signaling in cerebral 

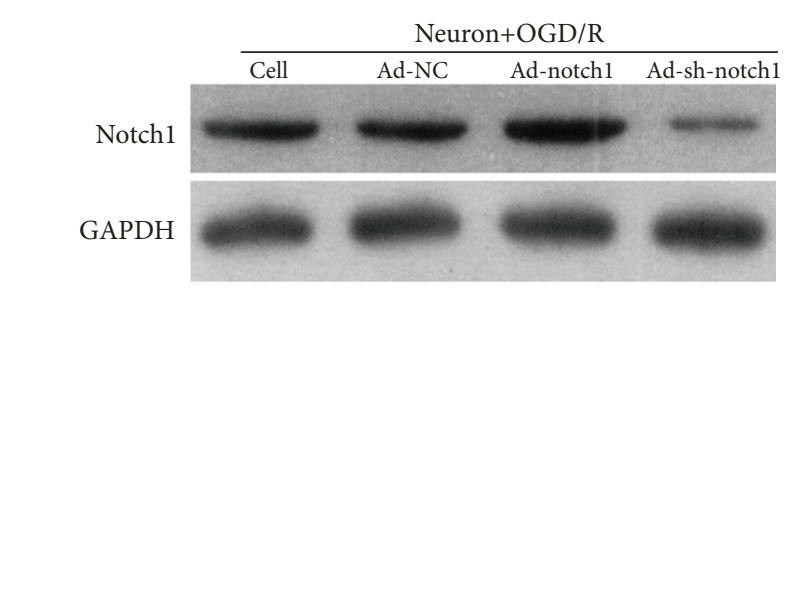

(a)

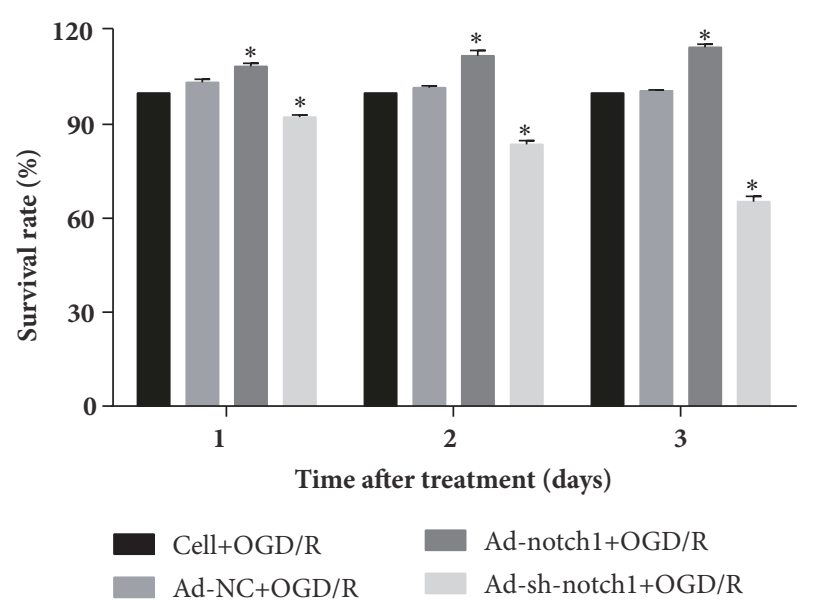

(b)
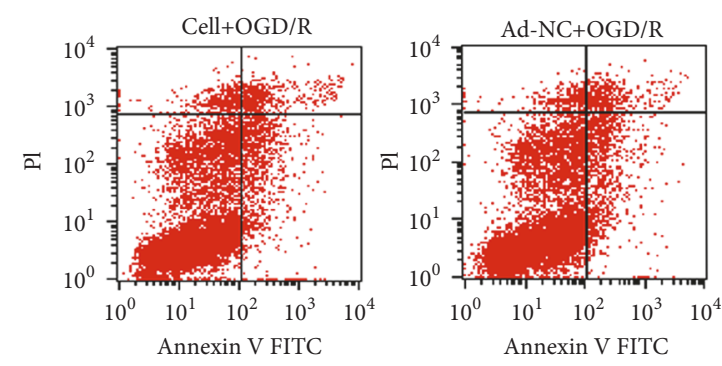

(d)
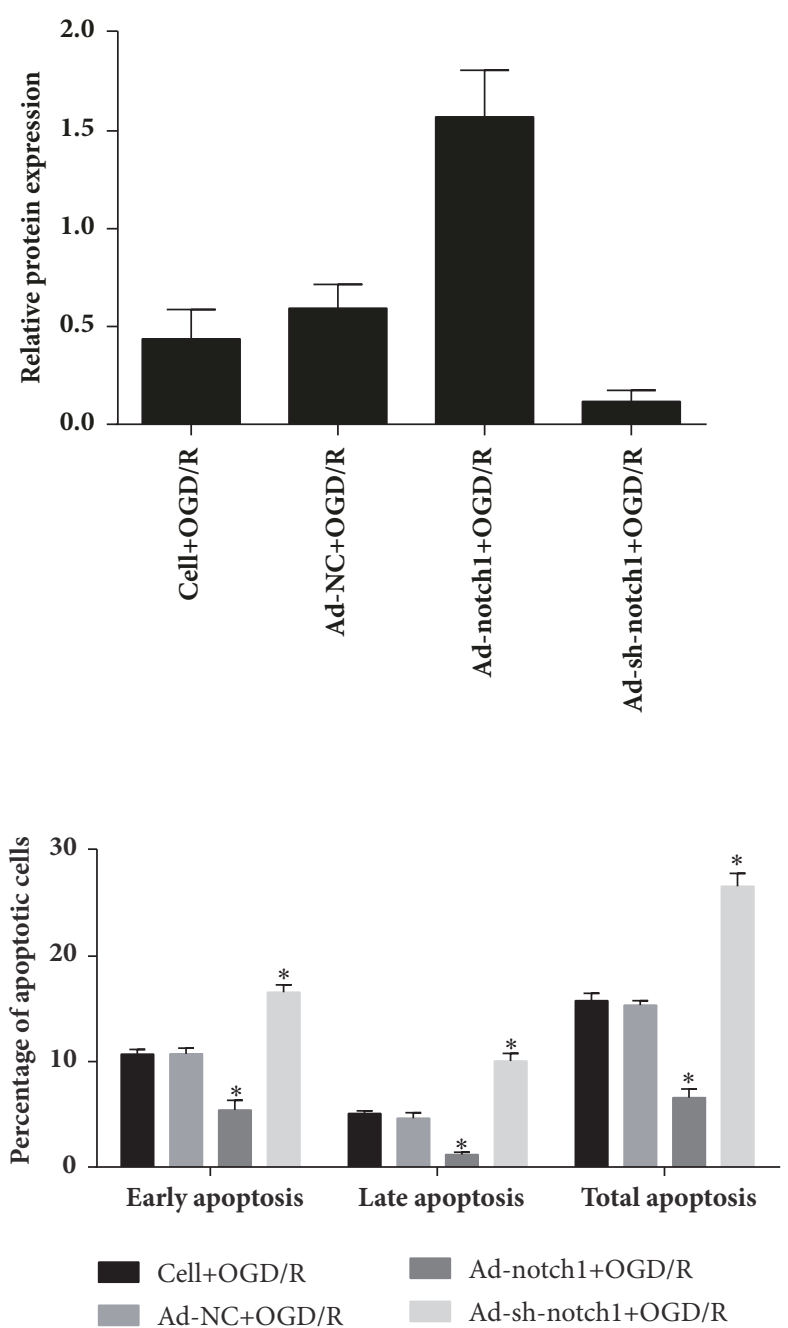

(c)


FIGURE 7: Effect of Notch1 overexpression and silencing on OGD/R-treated neuron cell survival and cellular apoptosis. (a) Notch1 expression in neuron was overexpressed or silenced by transfected Notchl overexpression adenovirus (Ad-Notch1) and Notch1 shRNA adenovirus (Adsh-Notch1). (b) The survival rate of each cell group was calculated by detecting the absorbance at $490 \mathrm{~nm}$ using the CellTiter 96 AQueous One Solution Cell Proliferation Assay Kit. (c-d) The percentage of apoptotic cells of each cell group was detected by flow cytometry analysis. (c) Statistical result of the percentage of apoptotic cells. (d) Represented images. ${ }^{*} p<0.05$.

$\mathrm{I} / \mathrm{R}$ injury and its possibility as a target for the treatment of cerebral I/R injury-related diseases. Nevertheless, more effort is needed to find the specific activator of the Notch signaling pathway to simulate the function of cIPC.
In conclusion, cIPC can activate and advance the peak expression of Notch signaling after $\mathrm{MCAO} / \mathrm{R}$. The neuroprotective effect of cIPC on cerebral I/R is diminished by blocking the Notch signal. These findings indicate that Notch 


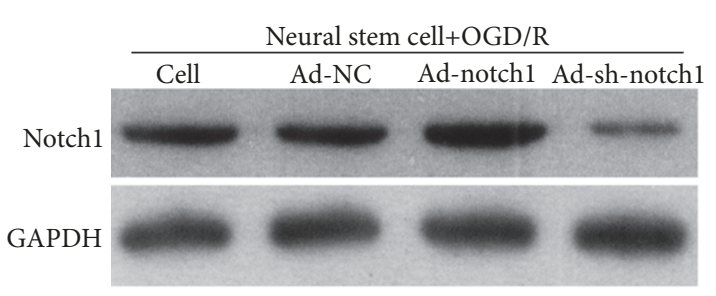

(a)


(b)

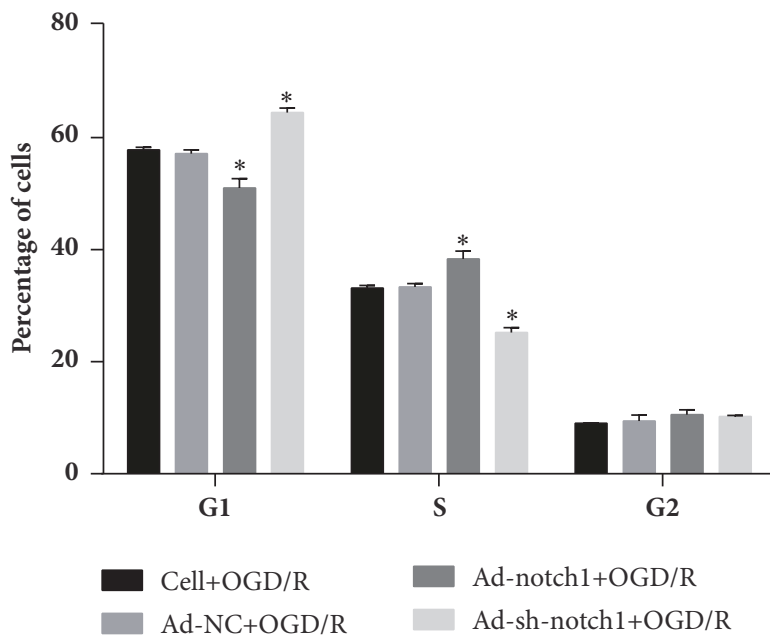

(c)

FIGURE 8: Effect of Notch1 overexpression and silencing on OGD/R-treated neural stem cell cycle. (a) The Notch1 expression in OGD/Rtreated neural stem cell was overexpressed or silenced by transfected Notchl overexpression adenovirus (Ad-Notchl) and Notch1 shRNA adenovirus (Ad-sh-Notch1). (b-c) The percentage of G1, S, and G2 stage cells of each cell group cell was detected by flow cytometry analysis. (b) Represented images. (c) Statistical result of the percentages of G1, S, and G2 stage cells. ${ }^{*} p<0.05$.

signals are crucial pathways for inducing ischemic tolerance by cIPC. This work establishes a foundation for future research to investigate new mechanisms and therapeutic targets of cerebral I/R injury-related diseases.

\section{Data Availability}

The data used to support the findings of this study are available from the corresponding author upon request.

\section{Conflicts of Interest}

The authors who have taken part in this study declare that they do not have anything to disclose regarding funding or conflicts of interest with respect to this paper.

\section{Authors' Contributions}

Li Chen, Kuan Huang, and Rong Wang contributed equally to this work. 


\section{Acknowledgments}

The work is supported by the National Natural Science Foundation of China (no. 81260202). The authors thank the members of their laboratories for their insight and technical support.

\section{References}

[1] M. Ashafaq, M. M. Khan, S. Shadab Raza et al., "S-allyl cysteine mitigates oxidative damage and improves neurologic deficit in a rat model of focal cerebral ischemia," Nutrition Research, vol. 32, no. 2, pp. 133-143, 2012.

[2] J. Su, J. Liu, X.-Y. Yan et al., "Cytoprotective effect of the UCP2SIRT3 signaling pathway by decreasing mitochondrial oxidative stress on cerebral ischemia-reperfusion injury," International Journal of Molecular Sciences, vol. 18, no. 7, 2017.

[3] H.-J. Steiger and D. Hänggi, "Ischaemic preconditioning of the brain, mechanisms and applications," Acta Neurochirurgica, vol. 149, no. 1, pp. 1-10, 2007.

[4] P. Meybohm, J. Renner, O. Broch et al., "Postoperative Neurocognitive Dysfunction in Patients Undergoing Cardiac Surgery after Remote Ischemic Preconditioning: A DoubleBlind Randomized Controlled Pilot Study," PLOS ONE, vol. 8, no. 5, Article ID e64743, 2013.

[5] S. Koch, M. Katsnelson, C. Dong, and M. Perez-Pinzon, "Remote ischemic limb preconditioning after subarachnoid hemorrhage: a phase Ib study of safety and feasibility," Stroke, vol. 42, no. 5, pp. 1387-1391, 2011.

[6] D. J. Hausenloy, P. K. Mwamure, V. Venugopal et al., "Effect of remote ischaemic preconditioning on myocardial injury in patients undergoing coronary artery bypass graft surgery: a randomised controlled trial," The Lancet, vol. 370, no. 9587, pp. 575-579, 2007.

[7] M. Thielmann, E. Kottenberg, P. Kleinbongard et al., "Cardioprotective and prognostic effects of remote ischaemic preconditioning in patients undergoing coronary artery bypass surgery: a single-centre randomised, double-blind, controlled trial," The Lancet, vol. 382, no. 9892, pp. 597-604, 2013.

[8] R. Zhang, A. Engler, and V. Taylor, "Notch: an interactive player in neurogenesis and disease," Cell and Tissue Research, vol. 371, no. 1, pp. 73-89, 2017.

[9] T. J. Pierfelice, K. C. Schreck, C. G. Eberhart, and N. Gaiano, "Notch, neural stem cells, and brain tumors," Cold Spring Harbor Symposium on Quantitative Biology, vol. 73, pp. 367-375, 2008.

[10] J. Guan, X. Wei, S. Qu, T. Lv, Q. Fu, and Y. Yuan, "Osthole prevents cerebral ischemia-reperfusion injury via the Notch signaling pathway," The International Journal of Biochemistry \& Cell Biology, vol. 95, no. 4, pp. 459-467, 2017.

[11] K. Yoon and N. Gaiano, "Notch signaling in the mammalian central nervous system: insights from mouse mutants," Nature Neuroscience, vol. 8, no. 6, pp. 709-715, 2005.

[12] H.-P. Zhang, Y.-Y. Sun, X.-M. Chen et al., “The neuroprotective effects of isoflurane preconditioning in a murine transient global cerebral ischemia-reperfusion model: The role of the notch signaling pathway," NeuroMolecular Medicine, vol. 16, no. 1, pp. 191-204, 2014.

[13] N. Gaiano and G. Fishell, "The role of Notch in promoting glial and neural stem cell fates," Annual Review of Neuroscience, vol. 25, pp. 471-490, 2002.
[14] E. Z. Longa, P. R. Weinstein, S. Carlson, and R. Cummins, "Reversible middle cerebral artery occlusion without craniectomy in rats," Stroke, vol. 20, no. 1, pp. 84-91, 1989.

[15] M. L. Seibenhener and M. W. Wooten, "Isolation and culture of hippocampal neurons from prenatal mice," Journal of Visualized Experiments, no. 65, 2012.

[16] X. Wang, X. Wei, W. Zheng et al., "Study on in vitro isolation and culture method of neural stem cells from fetal rat neocortex," Journal of Hygiene Research, vol. 39, no. 6, pp. 674-677, 2010.

[17] X.-Q. Liu, R. Sheng, and Z.-H. Qin, “The neuroprotective mechanism of brain ischemic preconditioning," Acta Pharmacologica Sinica, vol. 30, no. 8, pp. 1071-1080, 2009.

[18] L. Alberi, S. E. Hoey, E. Brai, A. L. Scotti, and S. Marathe, "Notch signaling in the brain: in good and bad times," Ageing Research Reviews, vol. 12, no. 3, pp. 801-814, 2013.

[19] P. Balaganapathy, S. Baik, K. Mallilankaraman, C. G. Sobey, D. Jo, and T. V. Arumugam, "Interplay between Notch and p53 promotes neuronal cell death in ischemic stroke," Journal of Cerebral Blood Flow \& Metabolism, vol. 1, no. 10, 2017.

[20] A. Joutel and E. Tournier-Lasserve, "Notch signalling pathway and human disease," Seminars in Cell \& Developmental Biology, vol. 9, no. 6, pp. 619-625, 1998.

[21] Y. Zhang, J. B. Lian, J. L. Stein, A. J. Van Wijnen, and G. S. Stein, "The Notch-responsive transcription factor Hes-1 attenuates osteocalcin promoter activity in osteoblastic cells," Journal of Cellular Biochemistry, vol. 108, no. 3, pp. 651-659, 2009.

[22] Y.-L. Cheng, J.-S. Park, S. Manzanero et al., "Evidence that collaboration between HIF- $1 \alpha$ and Notch-1 promotes neuronal cell death in ischemic stroke," Neurobiology of Disease, vol. 62, pp. 286-295, 2014.

[23] T. V. Arumugam, Y.-L. Cheng, Y. Choi et al., "Evidence that $\gamma$ secretase-mediated notch signaling induces neuronal cell death via the nuclear factor- $\kappa \mathrm{B}-\mathrm{Bcl}-2$-interacting mediator of cell death pathway in ischemic stroke," Molecular Pharmacology, vol. 80, no. 1, pp. 23-31, 2011.

[24] S.-H. Baik, M. Fane, J. H. Park et al., "Pinl promotes neuronal death in stroke by stabilizing notch intracellular domain," Annals of Neurology, vol. 77, no. 3, pp. 504-516, 2015.

[25] T. V. Arumugam, S. L. Chan, D.-G. Jo et al., "Gamma secretasemediated Notch signaling worsens brain damage and functional outcome in ischemic stroke," Nature Medicine, vol. 12, no. 6, pp. 621-623, 2006.

[26] T. V. Arumugam, S. H. Baik, P. Balaganapathy, C. G. Sobey, M. P. Mattson, and D. G. Jo, "Notch signaling and neuronal death in stroke," Progress in Neurobiology, vol. 21, no. 17, pp. 30139-30139, 2018.

[27] Q. Yang, W. Yan, X. Li et al., "Activation of canonical notch signaling pathway is involved in the ischemic tolerance induced by sevoflurane preconditioning in mice.", Anesthesiology, vol. 117, no. 5, pp. 996-1005, 2012. 


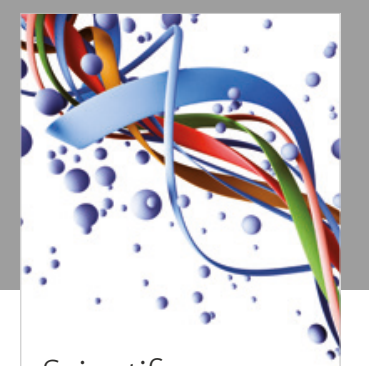

Scientifica
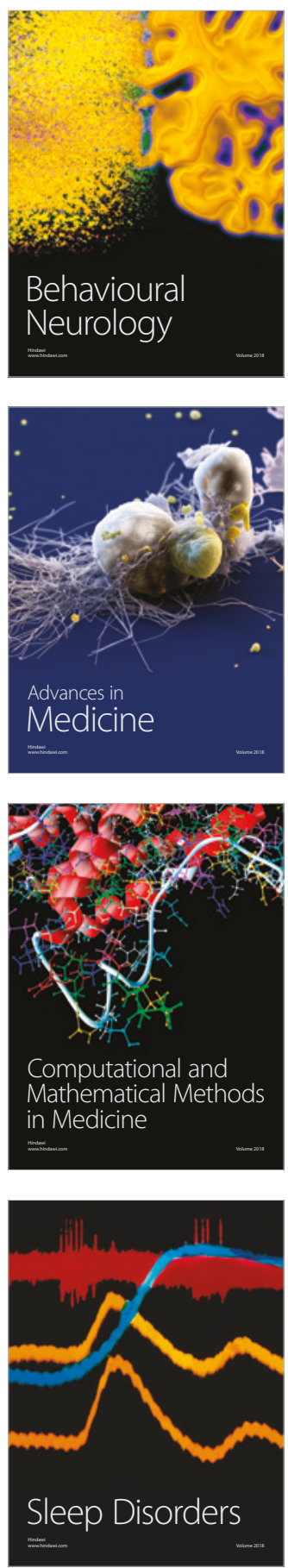

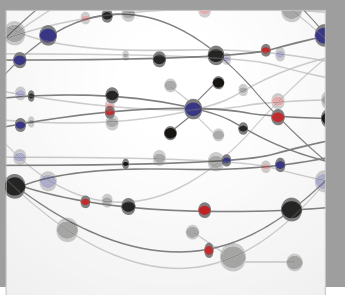

The Scientific World Journal

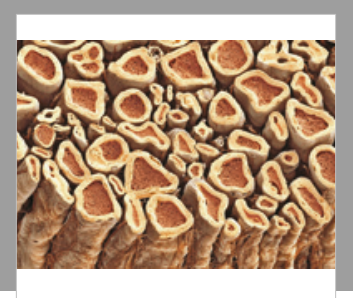

Case Reports in

Neurological Medicine

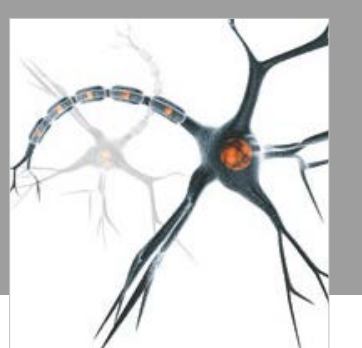

Neural Plasticity

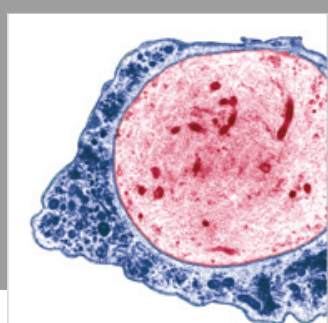

Multiple Sclerosis

International

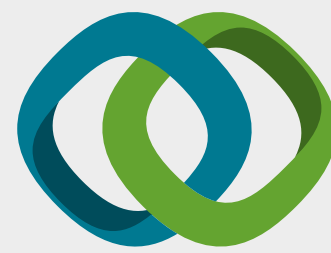

Hindawi

Submit your manuscripts at

www.hindawi.com


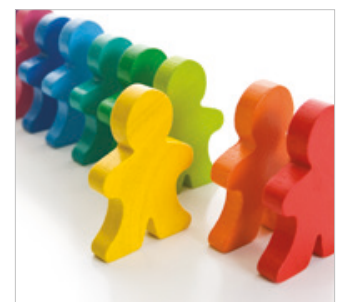

Autism

Research and Treatment
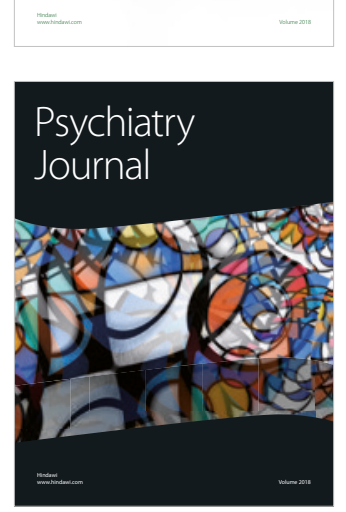
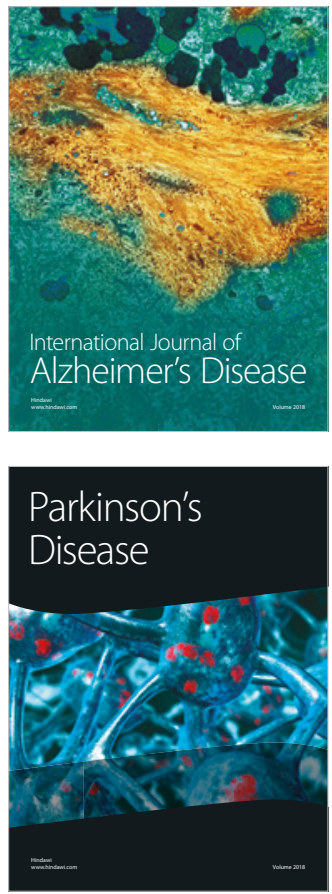
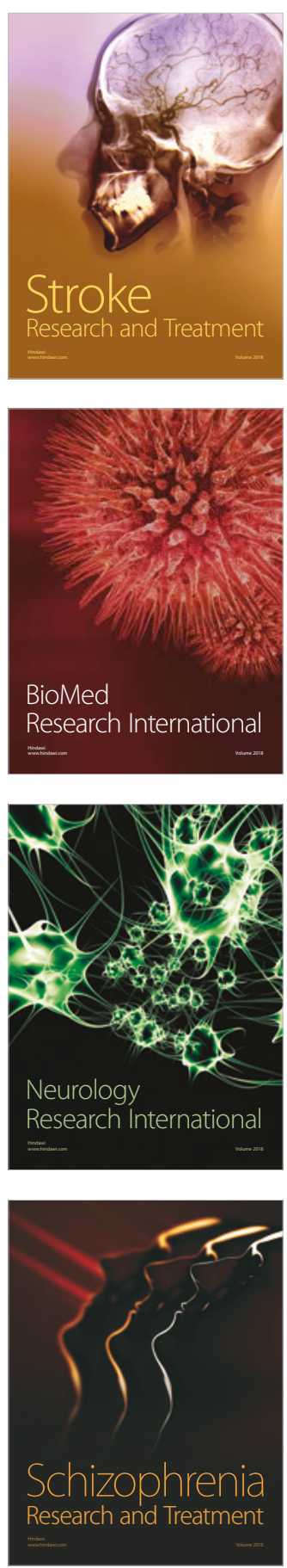